\title{
Efficacy and safety of dabigatran for venous thrombosis prophylaxis after knee replacement surgery in Thai patients: a prospective non-randomized controlled trial
}

Tawan Intiyanaravut ${ }^{1, *}$, Nimit Thongpulsawasdi', Napol Sinthuvanich', Sasikarn Songtaweesap²

Abstract

Background: Dabigatran, a direct oral anticoagulant, has been approved for the prevention of venous thromboembolism (VTE) after orthopedic surgery in many countries. The efficacy and safety of this agent were most studied in Western countries. Objective: To assess the efficacy and safety of dabigatran in preventing venous thromboembolic diseases after total knee arthroplasty (TKA) in Asian patients.

Methods: We conducted a prospective nonrandomized controlled study in Thai patients undergoing TKA. Thirtytwo patients received $220 \mathrm{mg}$ of dabigatran once daily for 14 days as thromboprophylaxis and 32 patients in the control group received none. The primary efficacy outcome was deep vein thrombosis (DVT), which was identified by color Doppler ultrasonography and/or diagnosed pulmonary embolism (PE). The primary safety outcomes were major bleeding and clinically relevant nonmajor bleeding events.

Results: There were no DVTs or PEs diagnosed in either group. The difference in the composite incidence of major and clinically relevant nonmajor bleedings between the dabigatran and control groups did not reach significant $(6.2 \%$ vs. $0 \%, P=0.15$ ).

Conclusion: Dabigatran might have no clear benefit for the prevention of VTE after TKA in Thai patients. We do not recommend the routine use of dabigatran as a chemical thromboprophylaxis after TKA in Thai patients. To determine the safety profile, further study with larger sample sizes is required.

Keywords: Asians; dabigatran; deep vein thrombosis; knee arthroplasty; prophylaxis

One major complication of total knee arthroplasty (TKA) is venous thromboembolism (VTE) [1]. The presentation of VTE could be asymptomatic deep vein thrombosis (DVT), symptomatic DVT, or pulmonary embolism (PE). Routine pharmacological thromboprophylaxis is recommended by the American
College of Chest Physicians [2]. In Asian countries, there is still a controversy about the routine use of VTE prophylaxis because of substantial variation in the reported incidences of VTE after TKA [3-7]. For example, the Thai conference on VTE in hip and knee surgery, held in 2015 , recommended the

*Correspondence to: Tawan Intiyanaravut, Department of Orthopedic Surgery, Golden Jubilee Medical Center, Faculty of Medicine Siriraj Hospital, Mahidol University, Nakhon Pathom 73170, Thailand, e-mail: tawan.int@mahidol.ac.th

'Department of Orthopedic Surgery, Golden Jubilee Medical Center, Faculty of Medicine Siriraj Hospital, Mahidol University, Nakhon Pathom 73170, Thailand

2Department of Radiology, Golden Jubilee Medical Center, Faculty of Medicine Siriraj Hospital, Mahidol University, Nakhon Pathom 73170, Thailand

¿ Open Access. ๑ 2019 Intiyanaravut et al., published by Sciendo. (ख्c) BY-NC-ND This work is licensed under the Creative Commons Attribution NonCommercial-NoDerivatives 4.0 License. 
use of chemoprophylaxis for VTE in Thai patients undergoing total joint arthroplasty only in high-risk patients due to bleeding complications. However, the increasing awareness of the risk of VTE after TKA in Asian patients and the development of direct oral anticoagulants (DOACs) has been associated with a reconsideration of VTE prophylaxis.

Dabigatran, a direct thrombin inhibitor, has been approved as an orally administered anticoagulant in Thailand since 2009 , and one of the approved indications is the prevention of VTE after orthopedic surgery. The efficacy of dabigatran in the prevention of VTE after hip and knee arthroplasty is comparable to conventional subcutaneous enoxaparin and has a similar bleeding profile in a pooled analysis of three trials [8]. However, the study locations were all in Western countries; there are few studies in Asian countries on dabigatran, one study from Japan showed the benefits of dabigatran in preventing VTE after TKA with a good safety profile [9].

We, therefore, conducted this prospective controlled study to evaluate the risks and benefits of venous thromboprophylaxis with dabigatran in Thai patients who underwent primary TKA.

\section{Materials and methods}

This was an investigator blind, single-center, prospective, nonrandomized, paralled-group, controlled study. The study protocol was approved by Mahidol University Ethics Committees (certificate of approval no. MU-IRB2012/169.1810), and all eligible patients signed written informed consent forms. The study has been registered at the Thai Clinical Trials Registry (TCTR20150504001).

Between May 2015 and December 2016, there were 152 total knee replacements performed in 150 patients in our center. Inclusion criteria were age between 18 and 90 years and primary elective total knee replacement. Exclusion criteria were any abnormal bleeding history within 1 year, a history of DVT or PE, a history of hemorrhagic stroke or gastrointestinal bleeding, treatment with anticoagulants or antiplatelet within 7 days before surgery, severe liver disease, chronic kidney disease stages 4 and 5, women of child-bearing potentials, and active malignant disease. A total of 150 patients met our inclusion criteria. Twelve patients were excluded from the study: four with chronic kidney disease staged 4 and 5 , three with previous use of anticoagulants, three with a history of hemorrhagic stroke or gastrointestinal bleeding, and two with a history of the previous DVT. Of the 138 eligible patients, 74 refused to participate, leaving 64 enrolled patients. We informed all enrolled patients about the study protocol as well as the risks and benefits of dabigatran and allowed the patients to decide independently whether or not to receive the prophylactic agent. Thirty-two patients who decided to receive oral dabigatran were included in the study group, whereas another 32 patients who refused to receive dabigatran were included in the control group. All 64 patients were studied prospectively. Sixty-two patients were diagnosed with primary osteoarthritis of the knee and two diagnosed with rheumatoid knee arthritis. Primary TKA was performed by three orthopedic surgeons. All patients received regional anesthesia with an adductor canal nerve block or femoral nerve block combined with spinal anesthesia. All prostheses were cemented, fixed bearing, and posterior stabilized knees (NexGen; LPS, LPSflex, Gender Solutions; Zimmer, Warsaw, Indiana). We used the intramedullary guide for the femoral cut and extramedullary guide for the tibial cut. One dose of antibiotic prophylaxis with Cefazolin or Clindamycin was given before the operation. The operated leg was exsanguinated before inflation of the tourniquet with over $150 \mathrm{mmHg}$ systolic blood pressure. The postoperative protocol included cold compression, compressive dressing in the first $24 \mathrm{~h}$, drain removal in $48-72 \mathrm{~h}$ after operation, and continuous passive movements initiated on the second day followed by active mobilization and full weight-bearing ambulation.

All patients in the study group received a single dose of oral dabigatran $110 \mathrm{mg}$ in the first postoperative day and then $220 \mathrm{mg}$ once daily for 14 days. The control group received no chemoprophylaxis. The patient demographic data, preoperative functional knee score (Oxford Knee Score), operative data (operative time, intraoperative blood loss), lengths of stay, and complications were recorded.

\section{Outcomes assessment}

The primary efficacy outcome was the cumulative incidence of VTE (ultrasound detectable DVT or clinical diagnosis of PE which confirmed by $\mathrm{CT}$ angiogram) during hospitalization. The ultrasonography was performed in all patients between the sixth and the tenth days after surgery by two radiologists who were blinded to whether prophylaxis was used. The primary endpoint depended on a positive diagnosis of thrombosis or loss of compressibility of a vein or the presence of high clinical suspicious for PE which was objectively confirmed by computed tomography pulmonary angiogram (CTPA). Clinical signs of DVT and PE were also evaluated as the secondary efficacy outcome using a score ranging from 0 (low suspicion) to 5 (high suspicion) for DVT and score ranging from 0 to 3 (low to high suspicion) for PE (Table 1).

The primary safety outcomes were the cumulative incidence of major bleeding events which were defined as bleeding that was fatal or involved a critical organ or clinically 
Table 1. Deep-vein-thrombosis (DVT) and pulmonary embolism (PE) clinical signs scoring system

\begin{tabular}{ll}
\hline Clinical signs & Score \\
\hline Deep-vein-thrombosis & \\
Swelling (circumferential diameter increase $>2 \mathrm{~cm}$ ) & 1 point \\
Warmth & 1 point \\
Discolored skin & 1 point \\
Visible surface vein & 1 point \\
Positive Homan sign (Calf tenderness) & 1 point \\
Pulmonary embolism & 1 point \\
Sudden dyspnea or tachypnea & 1 point \\
Chest pain & 1 point \\
Cough of hemoptysis
\end{tabular}

overt bleeding leading to transfusion of two or more units of whole blood or packed cells, and clinically relevant nonmajor bleeding events which were defined as the presence of Grade III hematoma (requiring operative removal or delayed rehabilitation). The secondary safety outcomes were minor bleeding other than major bleeding or Grade II surgical site hematoma (hematoma extending to the calf, thigh, or popliteal region with hemarthrosis with no rehabilitation restriction), macroscopic hematuria, epistaxis, bleeding per gum, and skin bruise larger than $5 \mathrm{~cm}$ in diameter. Major, clinically relevant nonmajor and minor bleeding events were defined according to the European guideline [10]. Total blood loss was calculated from the sum of intraoperative blood loss and postoperative blood loss recorded from a drainage system. The total number of units of blood transfused was also evaluated. Clinical assessment of the surgical site hematoma was performed on the third and seventh days after surgery using a modified hematoma grading scale [11] from 0 to 3 (Table 2) to assess the extent of hematoma at the knee.

All patients were clinically evaluated or telephone interviewed between 3 and 6 months after surgery to evaluate late DVT, post-thrombotic syndrome, readmission of any reason, or other adverse events.

\section{Sample size calculation}

Literature reviews show the incidences of asymptomatic DVT after TKA without thromboprophylaxis in Asian patients ranges from $4 \%$ to $60 \%[4-6,12,13]$. With dabigatran as a thromboprophylaxis, the incidences of major VTE are approximately $4 \%$ [8]. We calculated the sample size based on the mean incidence of DVT in the control group at $32 \%$ and in the dabigatran group at $4 \%$, keeping an alpha error of 0.05 to give
Table 2. Hematoma grading score used for evaluation of hematoma and hemarthrosis

\begin{tabular}{lc}
\hline Description & Grade \\
\hline No hematoma or hemarthrosis & 0 \\
Minor hematoma around the wound with no hemarthrosis & I \\
Moderate hematoma around the wound extending to the \\
$\begin{array}{l}\text { calf, thigh, or popliteal region with hemarthrosis with no } \\
\text { rehabilitation restriction }\end{array}$ \\
$\begin{array}{l}\text { Severe hematoma or hemarthrosis threatening wound } \\
\text { healing, requiring knee aspiration, reoperation, or causing } \\
\text { delay rehabilitation }\end{array}$ \\
\hline
\end{tabular}

our study $80 \%$ of power and $10 \%$ of loss follow-up. Thus, we would need 32 patients in each group.

\section{Statistical analysis}

Intergroup comparison was determined using the Chi-squared test, Student's $t$-test, and Mann-Whitney-U test. Differences were considered significant when $P$ was $<0.05$.

\section{Results}

Between May 2015 and December 2016, 150 patients underwent TKA and were assessed for eligibility. There were 138 patients met all eligibility requirements. Among them, 74 patients refused to participate in this study and 64 patients were enrolled. Of these, 32 patients were included in the study group and 32 patients were included in the control group. All patients in both groups were evaluable for outcome assessment and completed the study (Figure 1). Intergroup differences were not significant for BMI, age, underlying comorbidity, preoperative knee score, and operative time (Table 3). However, the length of hospital stay for the control group was shorter than the study group ( 7.4 days vs. 8.4 days, $P=0.02$ )

\section{Thromboembolic events}

There was no ultrasound detectable DVT in both groups. No patients developed clinical signs or symptoms of PE. The mean DVT clinical scores are summarized in Table 4. No significant intergroup differences were found between the two groups.

\section{Bleeding-related events}

There was no patient in the control group who experienced major or clinically relevant bleeding complications $(0 \%)$. In the 


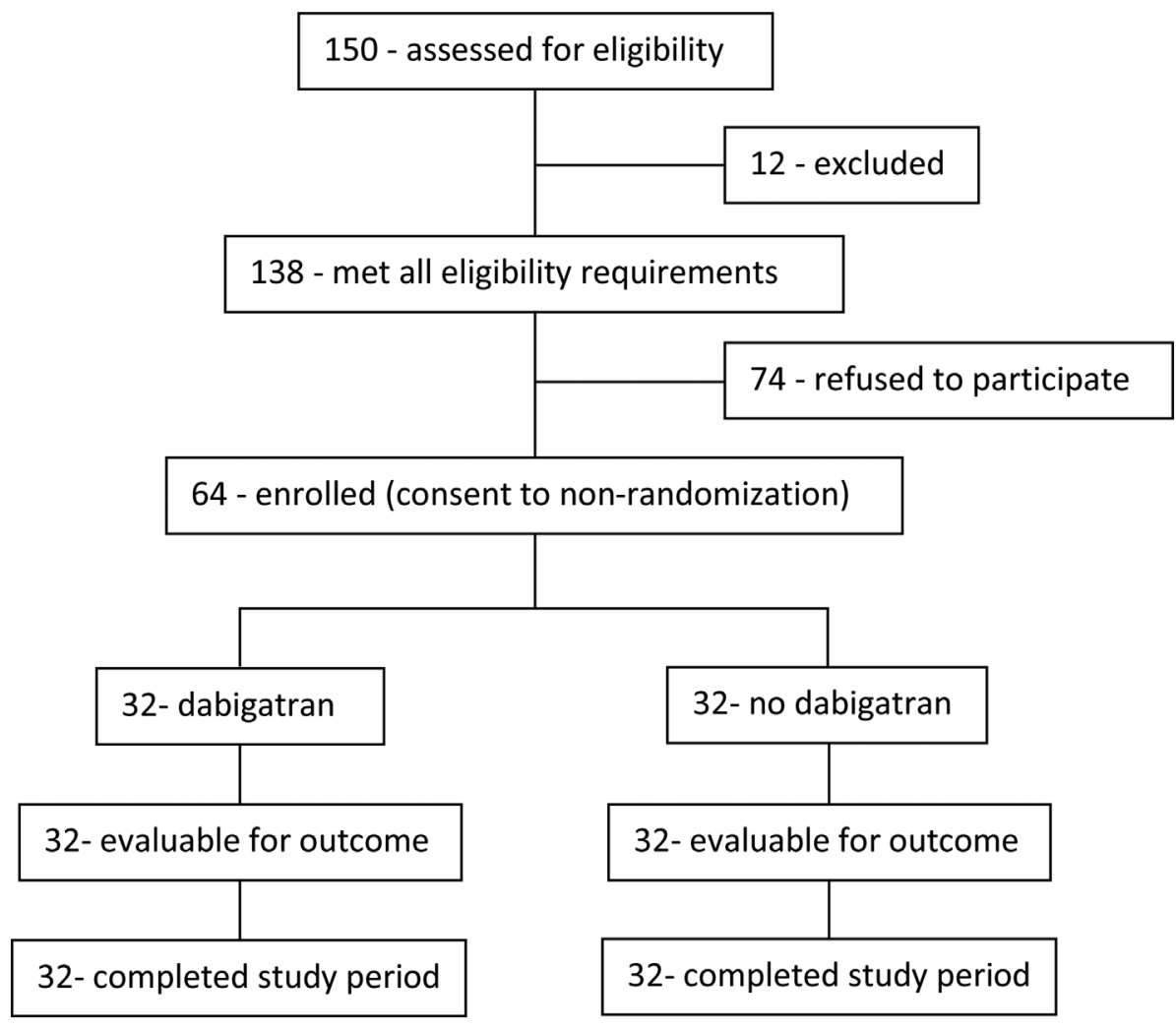

Figure 1. Progression of patients in the study

Table 3. Patient demographics data and operative characteristics

\begin{tabular}{lccc}
\hline Characteristic & $\begin{array}{c}\text { Control } \\
(\mathbf{N}=32)\end{array}$ & $\begin{array}{c}\text { Dabigatran } \\
\text { (N = 32) }\end{array}$ & $\boldsymbol{P}$ \\
\hline Age (years) & $68(7.2)$ & $69(7.4)$ & 0.63 \\
Women & $23(71 \%)$ & $27(84 \%)$ & 0.22 \\
Comorbidities & & & \\
HT & $20(62 \%)$ & $22(68 \%)$ & 0.59 \\
DM type 2 & $4(12 \%)$ & $4(12 \%)$ & 1 \\
Ischemic heart disease & $1(3 \%)$ & 0 & 0.31 \\
Cerebrovascular disease & $1(3 \%)$ & 0 & 0.31 \\
Previous history of inactive cancer & 0 & $1(3 \%)$ & 0.31 \\
Body mass index & $27.5(4.8)$ & $27.6(4.2)$ & 0.91 \\
Preoperative oxford knee score & $21.5(7.2)$ & $21.4(5.5)$ & 0.98 \\
Operative time (min) & $114.5(13.1)$ & $112.9(23.2)$ & 0.74 \\
Length of hospital stay (days) & $7.4(1.2)$ & $8.4(2.1)$ & 0.02 \\
\hline
\end{tabular}

Data are number (\%) or mean (SD).

dabigatran group, two patients had Grade III hemarthrosis at the surgical site; one of these patients needed four units of blood transfusion while the other required one unit (6.2\%). Minor bleeding events occurred in three patients $(9.3 \%)$ in the control group and four patients $(12.5 \%)$ in the dabigatran group $(P=0.68)$.
Table 4. Summary of the mean DVT clinical score

\begin{tabular}{lccc}
\hline DVT clinical score & $\begin{array}{c}\text { Control } \\
(\mathbf{N}=\mathbf{3 2})\end{array}$ & $\begin{array}{c}\text { Dabigatran } \\
(\mathbf{N}=\mathbf{3 2})\end{array}$ & $\boldsymbol{P}$ \\
\hline Mean DVT clinical score (SD) & & & \\
3 days after surgery & 0.28 & 0.31 & 0.63 \\
7 days after surgery & 0.21 & 0.18 & 0.95 \\
\hline
\end{tabular}

The details of bleeding events are summarized in Table 5. Total blood loss, total amount of blood transfusions, and the hematoma grading score are summarized in Table 6, and no significant differences were found between the two groups in all bleedingrelated events.

Two patients who had a Grade III surgical site hematoma required restriction of motion and delayed rehabilitation programs but did not require surgical intervention.

\section{Other observations}

There was one patient in the control group who had temporarily acute kidney injury on the first day after operation, and there was one patient in the treatment group who had an acute gout attack on the third day after operation. No other 
Table 5. Summary of details of bleeding complication

\begin{tabular}{|c|c|c|c|}
\hline Bleeding complications & $\begin{array}{l}\text { Control } \\
(N=32)\end{array}$ & $\begin{array}{l}\text { Dabigatran } \\
\qquad(N=32)\end{array}$ & $P$ \\
\hline Minor bleeding events, $n$ (\%) & $3(9.3)$ & $4(12.5)$ & 0.68 \\
\hline Macroscopic hematuria & 0 & 0 & \\
\hline Epistaxis & 0 & 1 & \\
\hline Skin bruise & 2 & 3 & \\
\hline Grade II hemarthrosis & 2 & 2 & \\
\hline \multicolumn{4}{|l|}{ Clinically relevant nonmajor bleeding } \\
\hline Grade III hemarthrosis, $n$ (\%) & $0(0)$ & $1(3.1)$ & 0.31 \\
\hline \multicolumn{4}{|l|}{ Major bleeding events } \\
\hline Major organ bleeding & $0(0)$ & $0(0)$ & 1 \\
\hline $\begin{array}{l}\text { Required more than two units blood } \\
\text { transfusion }\end{array}$ & $0(0)$ & $1(3.1)$ & 0.31 \\
\hline $\begin{array}{l}\text { The composite of major and clinically } \\
\text { relevant nonmajor bleeding events, } n(\%)\end{array}$ & $0(0)$ & $2(6.2)$ & 0.15 \\
\hline Total bleeding events, $n$ (\%) & $3(9.3)$ & $6(18.7)$ & 0.28 \\
\hline
\end{tabular}

Table 6. Summary of mean total blood loss, blood transfusion, and mean hematoma grading score

\begin{tabular}{lccl}
\hline Bleeding parameters & $\begin{array}{c}\text { Control } \\
(\boldsymbol{N}=\mathbf{3 2})\end{array}$ & $\begin{array}{c}\text { Dabigatran } \\
(\boldsymbol{N}=\mathbf{3 2})\end{array}$ & $\boldsymbol{P}$ \\
\hline Mean total blood loss in milliliter (SD) & $589(245)$ & $572(286)$ & 0.83 \\
Mean amount of blood transfusion in & $0.34(0.6)$ & $0.34(0.8)$ & 1.0 \\
unit (SD) & & & \\
Mean hematoma grading score (SD) & & & \\
Third day after surgery & $0.37(0.5)$ & $0.43(0.6)$ & 0.67 \\
Seventh day after surgery & $0.37(0.6)$ & $0.68(0.9)$ & 0.11 \\
\hline
\end{tabular}

peri-operative complications were observed in either group. At 3-6-month follow-up, no patients in either group had any signs of late DVT, post-thrombotic syndrome, or other surgical-related complications.

\section{Discussion}

There has been some controversy over the routine use of pharmacological prophylaxis for DVT after TKA in Asian countries because there is a substantial variation reported in the incidence of DVT in Asian centers [3-7]. Cohen [14], on behalf of the Asia-Pacific Thrombosis Advisory Board, reviewed the incidence of DVT from local Asian studies including eight countries. The incidence of DVT ranged from 0 to $81.3 \%$; he concluded that current guideline recommendations should be implemented in Asia for the routine use of postoperative thromboprophylaxis. In contrast, in Thailand, the Thai conference on VTE in hip and knee surgery, held in September 2015, recommended to use chemoprophylaxis for VTE in Thai patients undergoing total joint arthroplasty only in the high-risk because of the risk of bleeding complications.

Dabigatran, a direct thrombin inhibitor, has been approved as an orally administrated anticoagulant in many countries including Thailand. Food and Drug Administration (FDA) of Thailand has approved dabigatran since 2009 for the prevention of stroke and VTE in atrial fibrillation (AF), orthopedic surgery, and treatment of DVT and PE. Recently, dabigatran has been widely accepted as chemoprophylaxis after orthopedic operations because it is convenient to administer, does not need coagulation monitoring and has comparable efficacy for venous thromboprophylaxis to enoxaparin [8]. However, the uncertainty in the safety profile may be a major limitation of DOACs in clinical practice [15].

For the efficacy outcome, we found no thromboembolic complication after TKA in Thai patients in both the control and the dabigatran groups. This finding supports our previous study that the incidence of thromboembolism after primary TKA in Thai patients is very low with or without chemical prophylaxis [16]. We used Doppler ultrasonography as the diagnostic tool for detecting DVT in all patients in this study because it was noninvasive and reliable; however, it was an operator-dependent method and had low sensitivity for the detection of asymptomatic DVT in the calf [17], which was a limitation in this study, however, we believe that asymptomatic distal DVT has low clinical significance and needs no treatment. Vaitkus et al. [18] studied the mortality rate of asymptomatic DVT in medical patients. He concluded that asymptomatic proximal DVT associated with increased mortality rate; however, no significant difference was observed in the mortality rate between the asymptomatic distal DVT and no DVT groups. We also used the mean DVT and PE clinical score as a secondary efficacy outcome measurement; however, no significant difference was observed between the two groups. We developed these scores in our recent study [16] to assess clinical signs of DVT and PE. The DVT clinical score had no correlation with the diagnosis of DVT, and there was no patient had clinical signs suspected for PE.

For safety outcome measurement, our study shows no significant difference in the composite of major and clinically relevant nonmajor bleeding events as well as minor bleeding events between the control group and the dabigatran group. Friedman et al. [8] reported the incidence of bleeding complications from a pooled analysis of three major trials (RE-MOBILIZE, 
RE-MODEL, and RE-NOVATE). Major bleeding incidence of dabigatran $220 \mathrm{mg}$ was $1.4 \%$ which was lower than in our study $(3.1 \%)$. The composite of major and clinically relevant bleeding event was 5.6\% compared with our finding (6.2\%) and the minor bleeding event of dabigatran $220 \mathrm{mg}$ was $6.6 \%$ which was lower than our finding (12.5\%). The difference in incidence between pooled data and our finding in minor bleeding events is because the criteria for minor bleeding events are different in details. The mean length of stay is longer in the dabigatran group than in the control group, 8.4 days vs. 7.4 days $(P<0.05)$ because one patient with Grade III surgical site hematoma in the dabigatran group had delayed rehabilitation program, and the length of stay was 17 days.

The findings in this study suggest that dabigatran has a questionable benefit-risk profile for the prevention of VTE after TKA Thai patients in general. However, the small number of the study population, in this study, could not show any statistically significant differences in all outcome measurements, which is a limitation in this study. We found that the calculated sample size based on the incidence of venous thromboembolic events from the past literature was higher than in our study because most studies used venography as the diagnostic tool. However, we used ultrasonography because the major advantages in terms of its noninvasive nature and the absent risk of thrombosis, skin irritation, and necrosis secondary to contrast injection required in venography. Grady-Benson et al. [19] demonstrated 100\% sensitivity, specificity, and accuracy of duplex ultrasonography compared with venography in detecting proximal DVT and $88 \%$ sensitivity, 98\% specificity, and 98\% accuracy in detecting distal DVT. Further studies with larger sample size are needed to confirm our finding.

\section{Conclusion}

We concluded that dabigatran might have no clear benefit for the prevention of VTE after TKA in Thai patients. We do not recommend the routine use of dabigatran as a chemical thromboprophylaxis after TKA in general Thai patients. High-risk patients for VTE should be considered for using chemical prophylaxis, depending on each patient's characteristics. Further studies, including a larger study population, are needed to confirm our findings.

Author contributions. TI contributed substantially to the conception and design of this study. NT, NS, and SS contributed substantially to the acquisition and interpretation of the data. TI drafted the manuscript. All the authors contributed substantially to its critical revision, approved the final version submitted for publication, and take responsibility for the statements made in the published article.

Acknowledgment. We did not receive any specific grant for this research from any funding agency in the public, commercial, or not-for-profit sectors.

Conflict of interest statement. The authors have completed and submitted the International Committee of Medical Journal Editors Uniform Disclosure Form for Potential Conflicts of Interest. None of the authors disclose any conflict of interest. Dabigatran that used in this study was supported by Boehringer Ingelheim (Thai) Company Limited. No funding or other supports from Industry was received for this study.

\section{References}

[1] Stulberg BN, Insall JN, Williams GW, Ghelman B. Deep-vein thrombosis following total knee replacement. An analysis of six hundred and thirty-eight arthroplasties. J Bone Joint Surg Am. 1984; 66:194-201.

[2] Falck-Ytter Y, Francis CW, Johanson NA, Curley C, Dahl OE, Schulman S, et al. Prevention of VTE in orthopedic surgery patients: antithrombotic therapy and prevention of thrombosis, 9th ed. American College of Chest Physicians Evidence-Based Clinical Practice Guidelines. Chest. 2012; 141:e278S-e325S.

[3] Bin Abd Razak HR, Soon AT, Dhanaraj ID, Tan AH. Incidence of clinically significant venous thromboembolic events in Asian patients undergoing total knee arthroplasty without anticoagulation. J Arthroplasty. 2012; 27:1128-32.

[4] Kim YH, Kim JS. Incidence and natural history of deep-vein thrombosis after total knee arthroplasty. A prospective, randomised study. J Bone Joint Surg Br. 2002; 84:566-70.

[5] Ko PS, Chan WF, Siu TH, Khoo J, Wu WC, Lam JJ. Deep venous thrombosis after total hip or knee arthroplasty in a low-risk Chinese population. J Arthroplasty. 2003; 18:174-9.

[6] Leizorovicz A, Committee SVSS (SMART Venography Study Steering Committee). Epidemiology of post-operative venous thromboembolism in Asian patients. Results of the SMART venography study. Haematologica. 2007; 92:1194-200.

[7] Won MH, Lee GW, Lee TJ, Moon KH. Prevalence and risk factors of thromboembolism after joint arthroplasty without chemical thromboprophylaxis in an Asian population. J Arthroplasty. 2011; 26:1106-11.

[8] Friedman RJ, Dahl OE, Rosencher N, Caprini JA, Kurth AA, Francis CW, et al. Dabigatran versus enoxaparin for prevention of venous thromboembolism after hip or knee arthroplasty: a pooled analysis of three trials. Thromb Res. 2010; 126:175-82.

[9] Fuji T, Fuijita S, Ujihira T, Sato T. Dabigatran etexilate prevents venous thromboembolism after total knee arthroplasty in Japanese patients with a safety profile comparable to placebo. J Arthroplasty. 2010; 25:1267-74.

[10] The European Agency for the Evaluation of Medicinal Products, Committee for Medicinal Products for Human Use (CHMP). 
Guideline on clinical investigation of medicinal products for prophylaxis of high intra- and post-operative venous thromboembolic risk. [online] 2012. [cited 2012 Mar 15]. Available from: https://www.ema.europa.eu/en/documents/scientific-guideline/ guideline-clinical-investigation-medicinal-products-prophylaxis-highintra-post-operative-venous_en.pdf.

[11] Blanchard J, Meuwly JY, Leyvraz PF, Miron MJ, Bounameaux H, Hoffmeyer P, et al. Prevention of deep-vein thrombosis after total knee replacement. Randomised comparison between a low-molecularweight heparin (nadroparin) and mechanical prophylaxis with a foot-pump system. J Bone Joint Surg Br. 1999; 81:654-9.

[12] Nathan S, Aleem MA, Thiagarajan P, Das De S. The incidence of proximal deep vein thrombosis following total knee arthroplasty in an Asian population: a Doppler ultrasound study. J Orthop Surg (Hong Kong). 2003; 11:184-9.

[13] Sudo A, Sano T, Horikawa K, Yamakawa T, Shi D, Uchida A. The incidence of deep vein thrombosis after hip and knee arthroplasties in Japanese patients: a prospective study. J Orthop Surg (Hong Kong). 2003; 11:174-7.

[14] Cohen AT, Asia-Pacific Thrombosis Advisory Board. Asia-Pacific Thrombosis Advisory Board consensus paper on prevention of venous thromboembolism after major orthopaedic surgery. Thromb Haemost. 2010; 104:919-30.

[15] Rostagno C. New oral anticoagulants in prophylaxis of venous thromboembolic disease in major orthopedic surgery. Cardiovasc Hematol Disord Drug Targets. 2016; 15:204-9.

[16] Intiyanaravut T, Thongpulsawasdi N, Sinthuvanich N, Teavirat S, Kunopart M. Enoxaparin versus no anticoagulation prophylaxis after total knee arthroplasty in Thai patients: a randomized controlled trial. J Med Assoc Thai. 2017; 100:42-9.

[17] Barnes RW, Nix ML, Barnes CL, Lavender RC, Golden WE, Harmon $\mathrm{BH}$, et al. Perioperative asymptomatic venous thrombosis: role of duplex scanning versus venography. J Vasc Surg. 1989; 9:251-60.

[18] Vaitkus PT, Leizorovicz A, Cohen AT, Turpie AG, Olsson CG, Goldhaber SZ, et al. Mortality rates and risk factors for asymptomatic deep vein thrombosis in medical patients. Thromb Haemost. 2005; 93:76-9.

[19] Grady-Benson JC, Oishi CS, Hanson PB, Colwell CW Jr, Otis SM, Walker RH. Postoperative surveillance for deep venous thrombosis with duplex ultrasonography after total knee arthroplasty. J Bone Joint Surg Am. 1994; 76:1649-57. 